Professor A.V. Kiselev, one of the most respected Russian scientists and a member of our Editorial Advisory Board, died on July 17, 1984. In the October 1984 issue of Chromatographia we have already published an Eulogy in his memory (K. I. Sakodynskii and L. S. Ettre, Chromatographia 18, 541 (1984)).

Just before his death, Dr. Kiselev submitted to us a number of manuscripts reporting on his most recent research. We are publishing these papers in the present issue of our journal. On this occasion we have asked Professor Georges Guiochon to summarize the importance of Dr. Kiselev's work.

The Editors

\title{
The Achievements of Professor A. V. Kiselev in the Field of Adsorption and Chromatography
}

\author{
G. Guiochon* \\ Chemistry Department, Georgetown University, Washington, DC 20057, USA
}

C. Vidal-Madjar

Ecole Polytechnique, Palaiseau, France

Dr. A. V. Kiselev has made considerable contributions to the progress of our understanding of adsorption on solid surfaces, both in gas-solid and liquid-solid adsorption.

Dr. Kiselev realized very early that gas chromatography (GC) is a powerful method to study gas-solid adsorption. Research on adsorption and in the related fields has been traditionally very strong in the U.S.S.R., and as many good physical chemistry students in this country, Dr. Kiselev also began his research work in this field. The traditional methods of manometry and calorimetry have been widely used for such investigations. Shortly after World War II Dr. Kiselev pioneered in the use of other methods such as IR spectrometry and gas-solid chromatography (GSC). Since in GSC the adsorption or Henry constant is derived from the retention time of a small pulse, there is little relation between the amount of compound and the precision of the determination, as long as the signal-to-noise ratio is large enough. Given the sensitivity of GC detectors, it becomes easy to carry out measurements at surface coverages several orders of magnitude smaller than what is possible with the static methods for which the signal is proportional to sample size. Dr. Kiselev realized this fact very early and used it to study the heterogeneity of real surfaces. The Henry constants and the heats of adsorption at zero surface coverage obtained by GSC are different from the values extrapolated for a zero sample size from the results of the classical methods, usually quite larger, demonstrating the existence of a small amount of highenergy sites on most surfaces. Of all surfaces, graphitized thermal carbon black proved to be the most homogeneous one, the heat of adsorption being almost independent of the surface coverage. It is a non-polar high-energy surface whose properties have been largely studied by Kiselev.

Later Dr. Kiselev thought of using adsorption as a method of studying molecular structures; he called it chromatoscopy. Different structures for a given formula would result in different adsorption constants and $G C$ retention times. The first step was the derivation of a molecular theory of adsorption, permitting the calculations of the Henry constant for a given molecule. Because of the high homogeneity of the surface of graphitized thermal carbon black, and of its surface structure, Dr. Kiselev selected it as the reference solid. He showed that the adsorption potential can be calculated as the sum of the adsorption potential of all the possible pairs made with any atom of the graphite network and any atom of the studied molecule. Using a Lennard-Jones potential to describe this interaction and summing up for each atom of the molecule and a few thousand atoms of the carbon crystal, Kiselev could calculate the molecule's adsorption potential. Unfortunately, because he did not have enough computer power available he could not apply the classical rules of statistical thermodynamics to calculate the Henry constant, but he derived 
the principle of this calculation and showed experimentally the excellent correlation between adsorption potential and heat of adsorption. In numerous papers Kiselev and his coworkers studied the structure of hundreds of compounds, and showed how the method could be used to study details of the structure of organic compounds, including the height of the energy barrier which hinders free rotation around a single bond.

This method can also be used in a liquid mobile phase, and during these last years Kiseley was busy extending the applications of this method to liquid chromatography. His activities were partly hindered by the difficulties in preparing a suitable graphitized thermal carbon black material for HPLC.

With limited experiments, Kiselev has produced a huge amount of splendid experimental data. With little computer power, he made great progress in the theory of adsorption. He demonstrated to us the power of an approach to practical problems based on sound theoretical background. We only hope that the next generation of scientists will pick up and continue Dr. Kiselev's work, for the benefit of science. 